\title{
AN INFINITE FAMILY OF COUNTEREXAMPLES TO A CONJECTURE ON POSITIVITY
}

\author{
J. MIQUEL MARTÍNEZ
}

\begin{abstract}
Recently, G. Mason has produced a counterexample of order 128 to a conjecture in conformal field theory and tensor category theory in [Ma. Here we easily produce an infinite family of counterexamples, the smallest of which has order 72 .
\end{abstract}

If $G$ is a finite group and $\chi \in \operatorname{Irr}(G)$ is an irreducible complex character of $G$, we denote by $\nu_{2}(\chi)$ the Frobenius-Schur indicator of $\chi$. The main result of [Ma] is to produce an example of a finite group $G$ having an irreducible character $\chi$ such that $\nu_{2}(\chi)=1$, and $\chi^{2}$ contains an irreducible constituent $\psi$ with $\nu_{2}(\psi)=-1$. In this note, we produce an infinite number of examples.

Let $p$ be an odd prime. It is well known that $S=\mathrm{SL}_{2}(p)$ has a unique involution

$$
z=\left(\begin{array}{cc}
-1 & 0 \\
0 & -1
\end{array}\right)
$$

which inverts the elements of $V$. Let $V=C_{p} \times C_{p}$ be the natural module for $S$, and let $Q \leq S$ be any quaternion subgroup of order 8 . Recall that the unique irreducible character $\psi$ of $Q$ of degree 2 has Frobenius-Schur indicator $\nu_{2}(\psi)=-1$.

Theorem A. Let $G=V Q$ be the semidirect product. Let $1 \neq \lambda \in \operatorname{Irr}(V)$. Then $\lambda^{G}=\chi \in \operatorname{Irr}(G), \nu_{2}(\chi)=1$ and $\chi^{2}$ contains $\psi$.

Proof. Let $1 \neq \lambda \in \operatorname{Irr}(V)$. Notice that $\lambda^{z}=\bar{\lambda} \neq \lambda$. Hence if the stabilizer $G_{\lambda}>V$ then $Q_{\lambda}>1$ and $z \in Q_{\lambda}$, which is not possible. Thus $\lambda^{G}=\chi \in \operatorname{Irr}(G)$ by Problem 6.1 of [Is].

Now, if $g \in G$ then notice that $g^{2} \in V$ if and only if the order of $g V$ divides 2. This happens if and only if $g \in H=V\langle z\rangle$. Using that $\chi(g)=0$ if $g \notin V$ we have that

$$
\nu_{2}(\chi)=\frac{1}{|G|} \sum_{g \in G} \chi\left(g^{2}\right)=\frac{1}{|G|} \sum_{g \in G, g^{2} \in V} \chi\left(g^{2}\right)=\frac{1}{|G|} \sum_{g \in H} \chi\left(g^{2}\right) .
$$

If $v \in V$, notice that $(v z)^{2}=1$. Also $V=\left\{v^{2} \mid v \in V\right\}$, since $V$ has odd order. Therefore

$$
\nu_{2}(\chi)=\frac{1}{|G|}\left(|V| \chi(1)+|V|\left[\chi_{V}, 1_{V}\right]\right)=1
$$

2010 Mathematics Subject Classification. Primary 18D10, $20 \mathrm{C} 15$.

Key words and phrases. Fusion category, positivity conjecture, Frobenius-Schur indicator. 
Finally,

$$
\chi^{2}=\left(\left(\lambda^{G}\right)_{V} \lambda\right)^{G}=\left(\sum_{x \in Q} \lambda^{x} \lambda\right)^{G}
$$

contains $\left(\lambda^{z} \lambda\right)^{G}=\left(1_{V}\right)^{G}$, which contains $\psi$ and we are done.

\section{REFERENCES}

[Is] I. M. Isaacs, 'Character Theory of Finite Groups', AMS, Providence, 2008.

[Ma] G. Mason, 'A brief history of the positivity conjecture in tensor category theory', Bulletin of the Institute of Mathematics Academia Sinica (New Series), Vol. 14 (2019), No. 2 pp. $149-153$.

Department of Mathematics, Universitat de València, 46100 Burjassot, València, SPAIN

E-mail address: jomimar4@alumni.uv.es 